\title{
Brote de sarna en un hospital terciario a partir de un caso de sarna costrosa
}

\author{
Andrea Elgueta N., Yolanda Parada E., Wilma Guzmán G., Paula Molina C. y Patricia González A.
}

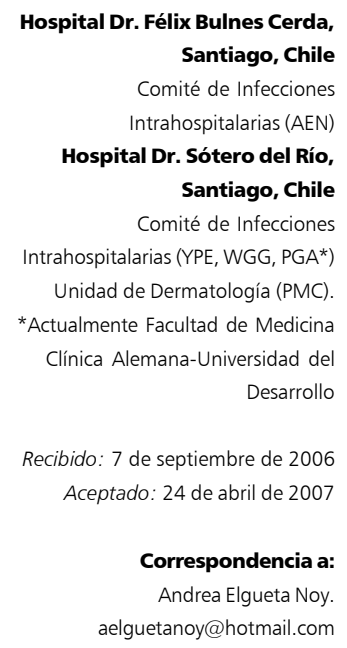

\section{An outbreak of scabies in a tertiary-care hospital from a crusted scabies case}

In February 2005 we performed an epidemiological study of an outbreak of scabies in a tertiary-care hospital which started from a crusted scabies case. We detected 10 secondary cases, 8 in healthcare workers and 2 in hospitalized patients. The attack rate was $4.1 \%$. In contrast to previously described outbreaks, the crusted scabies case was recognized at admission. The outbreak causes were: lacking adherence to contact precautions, long stay of the primary case in the hospital ward and delay of specific treatment. The main control measures were: alerting the hospital services about the outbreak, performing epidemiologic surveillance, coordinating with the Hospital Direction and the Occupational Health Department, education of healthcare workers in control measures, implementation of isolation measures and treatment of cases and contacts with $5 \%$ permethrin topical lotion.

Key words: scabies, crusted scabies, nosocomial outbreak, control of nosocomial outbreak.

Palabras claves: sarna, sarna costrosa, brote nosocomial, control de brote nosocomial.

\section{Introducción}

$\mathbf{L}$ a sarna o escabiosis es una ectoparasitosis cutánea de distribución universal, que afecta a personas de todas las edades y todos los estratos socio-económicos ${ }^{1}$. En Santiago de Chile, diferentes estudios han encontrado una prevalencia de 1 a $5 \%{ }^{2}$. El agente etiológico es el ácaro Sarcoptes scabiei var hominis, un parásito obligado que realiza su ciclo completo en la piel humana ${ }^{1}$. La hembra fecundada penetra la epidermis, formando galerías en las que deposita huevos y deyecciones. Las formas juveniles liberadas de los huevos migran a la superficie de la piel, siendo responsables de la transmisión de la enfermedad que ocurre, principalmente, por contacto directo pero también por contacto indirecto ${ }^{1,2}$. El período de incubación es de tres a seis semanas en la infestación primaria y de uno a tres días en las reinfestaciones ${ }^{1}$, ya que la expresión clínica es consecuencia de la infestación y de la hipersensibilidad frente a ésta ${ }^{2}$. La presentación clásica de la sarna es provocada por unos pocos ácaros ( 3 a 50) que se encuentran distribuidos en todo el cuerpo y se caracteriza por prurito intenso de predominio nocturno y lesiones típicas (surco acarino, vesículas perladas, nódulos escabióticos), principalmente, en las zonas de pliegues cutáneos. La forma noruega o costrosa es una infestación grave por el mismo ácaro, que se manifiesta en pacientes con algún grado de inmunocompromiso (SIDA, colagenopatías, síndrome de Down, quimioterapia, etc). Se caracteriza por hiperqueratosis y placas costrosas en las superficies no flexoras como son el tórax, cabeza, palmas y plantas; con prurito escaso. Esta forma de presentación es altamente transmisible debido a la gran cantidad de ácaros presentes (miles a millares) y, habitualmente, el diagnóstico se realiza en forma tardía por su baja frecuencia y sus manifestaciones atípicas; ambos factores facilitan el desarrollo de brotes epidémicos de sarna a partir de casos de sarna costrosa ${ }^{2,3}$.

A pesar de que la escabiosis es una enfermedad típicamente comunitaria, en el extranjero se han reportado múltiples brotes en recintos hospitalarios ${ }^{3-7} \mathrm{y}$ casas de reposo ${ }^{8,9}$, que han afectado a pacientes y a trabajadores de la salud. En Chile, no existen comunicaciones similares.

El Hospital Sótero del Río es un establecimiento de salud de nivel terciario que atiende a una población de 1.347.210 habitantes y cuenta con 700 camas. En febrero de 2005 se produjo un brote de sarna a partir de un paciente hospitalizado con sarna costrosa, que afectó a pacientes y al personal de salud. Los objetivos del presente estudio son describir el desarrollo de un brote de sarna en un hospital terciario, identificar los factores causales que desencadenaron el brote y evaluar el impacto de las medidas de control implementadas. 


\section{Pacientes y Método}

Se investigó, de manera prospectiva, un brote de sarna ocurrido en el Hospital Sótero del Río en febrero de 2005. La información se recolectó a través de visitas diarias a los servicios afectados, entrevistas verbales al personal de salud y revisión de fichas clínicas. Se vigiló la aparición de sarna en pacientes y personal de salud desde la admisión del caso primario ( 7 de febrero de 2005) hasta treinta días después ( 8 de marzo de 2005).

El periodo de duración del brote se consideró desde el ingreso del caso primario al hospital, hasta la pesquisa del último caso secundario. Se definió como caso sospechoso de sarna, a todo paciente hospitalizado o funcionario del establecimiento, con antecedente de haber estado en contacto (definido como el hecho de compartir unidad clínica) con el caso primario durante el período de brote, que desarrollara signos o síntomas de escabiosis. Se consideró caso confirmado de sarna, a todo caso sospechoso confirmado por la evaluación de un médico dermatólogo (no se realizó confirmación parasitológica de rutina).

\section{Resultados}

El período de duración de este brote fue de 21 días. El caso primario fue un paciente hombre, de 25 años de edad, portador de síndrome de Down y una psoriasis sin tratamiento, que consultó en la Unidad de Emergencia de Adultos el día 7 de febrero. Fue hospitalizado dos horas más tarde en la Unidad de Cuidados Intermedios (UCI) con diagnóstico de sepsis de posible foco dérmico y psoriasis extensa exacerbada, donde permaneció 12 horas, en sala compartida y bajo precauciones estándares. Al día siguiente se trasladó al Servicio de Medicina Interna, fue evaluado por dermatología y se realizó el diagnóstico de "sarna noruega", que fue confirmado por ácarotest. Se mantuvo al paciente en aislamiento de contacto y se postergó el tratamiento específico con loción de permetrina al 5\% por tener múltiples fisuras cutáneas e hiperqueratosis generalizada. Permaneció tres días en sala compartida siendo trasladado el 12 de febrero al Servicio de Especialidades, donde quedó, nuevamente, en sala compartida y en tratamiento con vaselina líquida. Siete días más tarde (19 de febrero) fue aislado en una sala individual, por cursar una bacteriemia por Acinetobacter baumannii multirresistente. Por este motivo, fue evaluado por el Comité de Infecciones Intrahospitalarias (CIIH) institucional, el que constató un cumplimiento insuficiente de las medidas de aislamiento de contacto por parte del personal y la postergación de la terapia específica. El tratamiento con loción de permetrina al $5 \%$, se inició el 25 de febrero, 17 días después de su ingreso, por no estar disponible en el hospital.

Casos secundarios: Se pesquisaron 10 casos de sarna, dos entre pacientes hospitalizados y ocho en el personal de salud; todos se presentaron dentro de tres semanas después del contacto con el caso primario (Figura 1). La tasa de ataque general del brote fue de $4,1 \%$, en un total de 224 expuestos (128 pacientes y 96 trabajadores de la salud) y la tasa de ataque por grupo fue de $1,6 \%$ en los pacientes y de $8,3 \%$ en el personal de salud. Los dos casos secundarios de pacientes pertenecían al Servicio de Especialidades y del personal de salud, siete correspondieron a técnicos paramédicos y uno a un auxiliar de servicio (Tabla 1).

Diagnóstico: Todos los casos sospechosos de sarna fueron confirmados por una evaluación dermatológica. No se realizó la confirmación de laboratorio de manera rutinaria.

Medidas de control: Una vez detectada la existencia del brote, se implementaron las siguientes medidas de control:

Aislamiento: El caso primario se mantuvo en una sala individual, con la puerta cerrada y bajo precauciones de transmisión por contacto, medidas sujetas a una supervisión diaria, hasta el alta. Los pacientes que fueron casos secundarios se mantuvieron en sala compartida, con precauciones de contacto hasta completar el tratamiento o el alta. Las precauciones de contacto incluyeron lavado de manos, antes y después de la

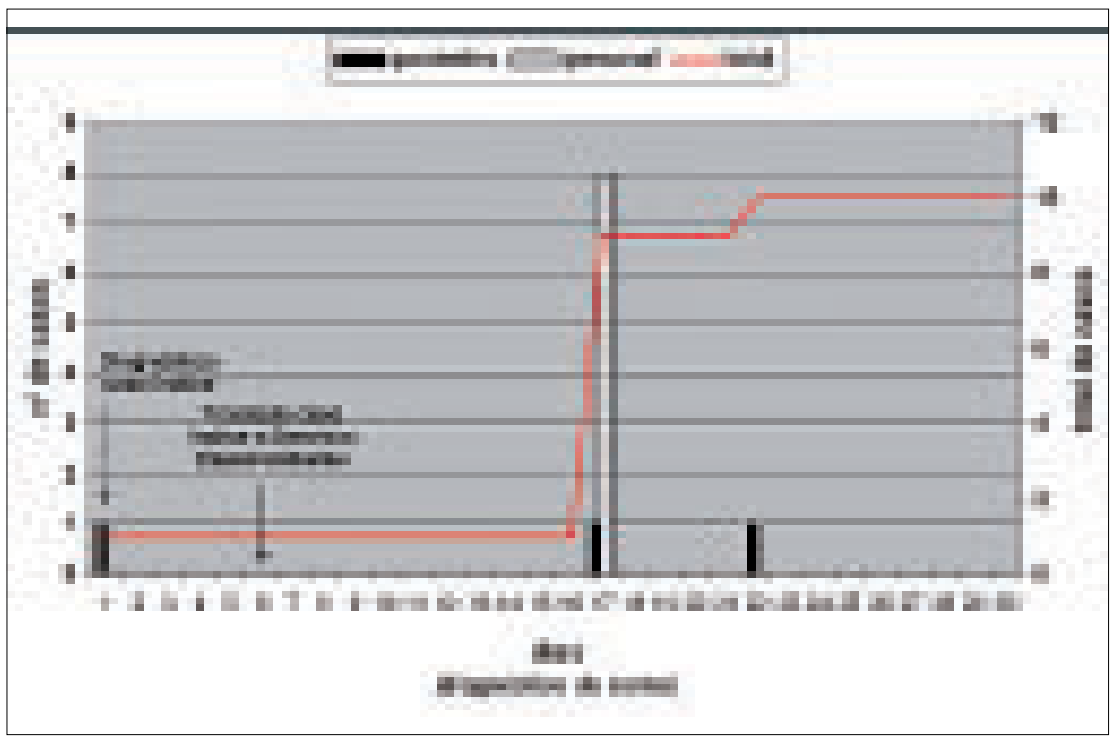

Figura 1. Curva epidémica del brote. 


\begin{tabular}{|c|c|c|c|c|c|c|c|}
\hline \multirow[t]{2}{*}{ Servicios clínicos } & \multirow{2}{*}{$\begin{array}{c}\text { Permanencia de caso } \\
\text { primario (días) }\end{array}$} & \multicolumn{3}{|c|}{$N^{\circ}$ casos secundarios $/ N^{\circ}$ expuestos } & \multicolumn{3}{|c|}{ Tasa ataque (\%) } \\
\hline & & TS & PH & Total & TS & PH & Total \\
\hline $\mathrm{UCl}$ & 0,5 & $0 / 24$ & $0 / 3$ & $0 / 27$ & 0 & 0 & 0 \\
\hline Medicina Interna & 3 & $0 / 55$ & $0 / 78$ & $0 / 133$ & 0 & 0 & 0 \\
\hline Especialidades & 20 & $8 / 17$ & $2 / 47$ & $10 / 64$ & 47,05 & 4,25 & 15,62 \\
\hline Total & 24 & $8 / 96$ & $2 / 128$ & $10 / 244$ & 8,33 & 1,56 & 4,09 \\
\hline
\end{tabular}

atención, uso de guantes no estériles y delantal protector, al entrar en contacto con el paciente o su unidad y artículos de uso clínico exclusivos para cada paciente (manguito de presión, termómetro, etc). Los trabajadores de la salud con sarna no requirieron licencia médica, pero fueron excluidos de la atención clínica hasta completar 24 horas de tratamiento.

Tratamiento: El caso primario se trató con loción de permetrina al $5 \%$ y vaselina azufrada en un esquema de tres días de permetrina, seguidos en cuatro días de vaselina azufrada, por dos veces. Los casos secundarios se trataron también con loción de permetrina al $5 \%$, por tres días seguidos, un intervalo libre de cuatro días y luego tres días más. Los contactos intrahospitalarios del caso primario del Servicio de Especialidades y los contactos intrafamiliares de los casos secundarios, fueron tratados con una aplicación de permetrina al $5 \%$.

Aseo y desinfección: La ropa de los pacientes con sarna fue manipulada con guantes y en bolsas cerradas rotuladas. El aseo de las salas y la limpieza de superficies se realizaron de rutina, de acuerdo con las normas del servicio.

Organización: Se realizó una reunión con la Dirección del hospital para informar acerca del brote y acordar las medidas de control y tratamiento que sería necesario instaurar. Además, se dio aviso al Departamento de Salud Ocupacional (DSO) del Servicio de Salud para coordinar el tratamiento del personal afectado y sus familias, ya que se consideró este contagio como una enfermedad profesional.

Capacitación: Mediante una reunión clínica, se capacitó a todo el personal del Servicio de Especialidades, en los mecanismos de transmisión de la enfermedad y las medidas de control del brote y tratamiento.

Vigilancia epidemiológica: Se informó a las jefaturas de todos los servicios en los que estuvo el caso primario de la existencia del brote, con el objetivo de detectar precozmente sintomatología compatible en pacientes o funcionarios. Se entrevistó, personalmente, a los funcionarios que atendieron directamente al caso primario, acerca de signos o síntomas compatibles con sarna. La vigilancia activa de nuevos casos de sarna se mantuvo hasta 30 días después de la hospitalización del caso índice.

\section{Discusión}

Se han reportado múltiples brotes de sarna en instituciones cerradas, siendo la causa más frecuente, la existencia de un paciente con sarna costrosa no diagnosticada a tiempo ${ }^{3,4,6,7}$, o sarna en pacientes postrados, ancianos o con trastornos mentales ${ }^{5,8,9}$. Estos últimos, requieren mayor apoyo de enfermería (por lo tanto contacto más estrecho con el personal), pueden presentar lesiones de distribución atípica $\mathrm{y}$, muchas veces, el prurito es atribuido a causas psicógenas o degenerativas, retrasando el diagnóstico de la enfermedad $^{5,8,9}$.

El caso primario de nuestra investigación fue un paciente con Síndrome de Down que ingresó al hospital con sarna costrosa. Sin embargo, a diferencia de lo reportado en otros brotes, el diagnóstico de la infestación se realizó el primer día de hospitalización. Consideramos que la transmisión al personal de salud y a otros pacientes en este caso, tuvo tres causas principales: falta de adherencia a las precauciones de transmisión por contacto, permanencia prolongada del caso primario en sala compartida (12 días desde el ingreso) y retardo en el inicio del tratamiento específico.

La tasa de ataque general fue de $4,1 \%$, bastante menor que la descrita en otros brotes ocurridos en instituciones similares ${ }^{4-6,9}$, en los que se han reportado tasas de 8,5 a $37 \%$. Probablemente, esto se deba a que, a pesar de la implementación tardía de las medidas de control de la sarna, la marcada alteración de la barrera cutánea del paciente y el conocimiento de su diagnós- 
tico al momento del ingreso, motivaron una mayor adherencia del personal de salud a las precauciones estándares. Las tasas de ataque por grupo confirman lo encontrado en otras publicaciones, evidenciando un mayor contagio de trabajadores de la salud que de pacientes $^{3,5,6}$, y dentro del primer grupo, mayor representación de los paramédicos que de los profesionales, ya que ellos mantienen un contacto más cercano y prolongado con los pacientes ${ }^{4}$. Todos los casos secundarios se presentaron en el Servicio de Especialidades, lugar donde el paciente permaneció hospitalizado más tiempo y donde, probablemente, el personal tiene menor entrenamiento en la técnica de aislamiento de contacto. La detección de los casos secundarios dentro de las tres primeras semanas después del contacto con el caso primario, coincide con lo descrito en otros brotes ${ }^{3,5,6}$.

Las medidas de control implementadas lograron la erradicación del brote. Consideramos que además de las recomendaciones internacionales para evitar la transmisión de sarna dentro del hospital ${ }^{10}$ (aislamiento de contacto con estricta supervisión de su cumplimiento, aseo y desinfección del ambiente, tratamiento de los casos), fueron fundamentales otras estrategias como: la comunicación directa y oportuna del $\mathrm{CIIH}$ con la Dirección del Hospital, el DSO y el personal de salud de los servicios afectados; la capacitación del personal en la prevención de la enfermedad y la administración profiláctica de permetrina a los contactos hospitalarios de mayor riesgo y familiares ${ }^{4}$. Esta última estrategia permitió cortar la cadena de transmisión precozmente y acortar el período de vigilancia activa de nuevos casos de sarna, a treinta días desde el ingreso del caso primario (sin considerar las seis semanas que puede durar el período de incubación desde el último caso diagnosticado).

La permetrina es el fármaco de elección en el tratamiento de la escabiosis ${ }^{1,4,11,12} \mathrm{y}$, en nuestra experiencia, fue efectiva en el tratamiento de todos los casos detectados en este brote y en la profilaxis. Es un acaricida potente, de baja absorción percutánea y baja toxicidad, por lo que se recomienda su uso, incluso, en pacientes prematuros, pacientes con daño neurológico $\mathrm{y}$ en fracasos de tratamiento con lindano ${ }^{12}$. El caso primario de este brote presentaba una psoriasis extensa además de la sarna noruega, con hiperqueratosis muy importante de algunas áreas y fisuras, que constituyeron el foco primario de una sepsis y determinó un retardo en el inicio del tratamiento específico. El uso de ivermectina oral se ha recomendado en la literatura para el tratamiento de sarna noruega, especialmente en pacientes que no toleran una terapia tópica, lo que podría haber evitado el retardo de la terapia específica en este caso. Sin embargo, este fármaco no está registrado ni disponible para uso humano en nuestro país (información aportada telefónicamente por el Centro Nacional de Información de Medicamentos y Farmacovigilancia, CENIMEF, del Instituto de Salud Pública de Chile) y su efectividad y seguridad requieren de mayor investigación ${ }^{1,11-13}$.

La identificación oportuna de los casos de sarna y la implementación precoz de medidas de control en instituciones cerradas son de gran importancia, ya que los brotes son difíciles de erradicar, pueden alcanzar períodos de duración prolongados (varios meses) y pueden asociarse a complicaciones infecciosas graves en pacientes debilitados.

Agradecimientos: A la Sra. Vivian Castro N, Terapeuta Ocupacional del Centro de Atención Integral del Funcionario (CAIF), del Departamento de Recursos Humanos del Servicio de Salud Metropolitano Sur Oriente, y a la Sra. Laudolina López C, funcionaria de la Unidad de Abastecimientos del Hospital Sótero del Río, por su valiosa ayuda en la recopilación de información.

\section{Resumen}

Realizamos el estudio epidemiológico de un brote de sarna ocurrido en un hospital terciario, a partir de un caso de sarna costrosa, en febrero de 2005. Detectamos diez casos secundarios; ocho en el personal de salud y dos en pacientes hospitalizados, con una tasa de ataque de $4,1 \%$. A diferencia de otros brotes, el diagnóstico de sarna costrosa se hizo al ingreso del caso primario al hospital. Las causas del brote fueron: adherencia deficiente a las medidas de aislamiento de contacto, permanencia prolongada del caso primario en sala compartida, y retardo en el inicio del tratamiento específico. Las principales medidas de control fueron: alertar a los servicios sobre el brote, realizar vigilancia epidemiológica, coordinación con la Dirección del Hospital y el Departamento de Salud Ocupacional, capacitar al personal de salud en las medidas de control, instaurar medidas de aislamiento y tratar a los casos y sus contactos con permetrina 5\% loción tópica. 


\section{Referencias}

1.- Chosidow O. Scabies. N Engl J Med 2006; 354: 1718-27.

2.- Saavedra T. Sarna y otras acarosis. Atías A. Parasitología Médica. $1^{\circ}$ Edición. Santiago de Chile: Ed Mediterráneo; 1999, p. 484-9.

3.- Zafar A, Beidas S, Silvestre L. Control of transmission of Norwegian scabies. Infect Control Hosp Epidemiol 2002; 23: 278-9.

4.- Obasanjo O, Wu P, Conlon M, Karanfil L, Pryor P, Moler G, et al. An outbreak of scabies in a teaching hospital: lessons learned. Infect Control Hosp Epidemiol 2001; 22: 13-8.

5.- Larrosa A, Cortés M, Martínez S, Clerencia C, Urdaniz L, Urbán J, et al. Nosocomial outbreak of scabies in a hospital in Spain. Euro Surveill 2003; 8: 199-203.

6.- Fajardo-Velázquez R, Urdez-Hernández E, Ysita-Morales A. Brote intrahospitalario de escabiosis, a partir de un caso de sarna noruega. Salud Pública Mex 2004; 46: 251-4.

7.- Jiménez-Lucho V, Fallon F, Caputo C, Ramsey K. Role of prolonged surveillance in the erradication of nosocomial scabies in an extended care veterans affairs medical center. Am J Infect Control 1995; 23: 44-9.

8.- De Beer G, Millar M, Tremblay L, Monette J. An outbreak of scabies in a log-term facility: the role of misdiagnosis and the costs associated with control. Infect Control Hosp Epidemiol 2006; 27: 517-8.

9.- Larrosa A, Cortés M, Clerencia C, Martínez S, Urdaniz L, Urbán J, et al. Brote de escabiosis en una residencia para personas mayores en la provincia de Huesca. Rev Esp Salud Pública 2004; 78: 107-14.

10.- Bolyard E, Tablan O, Williams W, Pearson M, Shapiro C, Deichtman S, et al. Guidelines for infection control in health care personnel. Am J Infect Control 1998; 26: 289-354.

11.- Walker G J A, Johnstone P W. Intervenciones para el tratamiento de la escabiosis (Revisión Cocharene traducida) http://www. cochrane.org/reviews/es/ab000320.html (accedido el 10 de agosto de 2006).

12.- Paasch U, Haustein U. Management of endemic outbreaks of scabies with allethrin, permethrin, and ivermectin. Int J Dermatol 2000; 39: 463-70.

13.- Scheinfeld N. Controlling scabies in institucional settings. Am J Clin Dermatol 2004; 5: 31-7. 